\title{
Thomas Pletschko
}

Medizinische Universität Wien

Clarissa Pelzer

Medizinische Universität Wien

\section{Gerda Rockenbauer}

Heilstättenschule Wien

\section{Agnes Turner}

Universität Klagenfurt

\section{Martin Röhsner}

Die Berater $\mathrm{GmbH}$

\section{Life happens wherever you are!}

\section{Einsatz des Avatars AV1 zur Verbesserung der schulischen Teilhabemöglichkeiten von Kindern und Jugendlichen mit chronischen Erkrankungen}

\section{DOI: https://doi.org/10.53349/sv.2021.i1.a66}

Kinder und Jugendliche mit chronischen Erkrankungen können aufgrund der Erkrankung und damit verbundenen Folgen oftmals die Schule nur unregelmäßig oder gar nicht besuchen. Um den negativen Folgeerscheinungen, welche mit längerer Schulabwesenheit einhergehen, entgegenzuwirken, werden in den letzten Jahren Telepräsenzsysteme als vielversprechender Ansatz diskutiert. In diesem Artikel wird ein Projekt der Medizinischen Universität Wien, der Universität Klagenfurt, der Heilstättenschule Wien sowie der Firma die Berater vorgestellt, welches sich mit der Frage beschäftigt, wie der Avatar AV1 als Telepräsenzsystem zur Verbesserung der schulischen und sozialen Teilhabemöglichkeiten von Kindern und Jugendlichen mit chronischen Erkrankungen beitragen kann. 


\section{Chronische Erkrankungen bei Kindern und Jugendlichen in Österreich}

Etwa 17.000 Kinder und Jugendliche in Österreich können aufgrund von medizinischen Behandlungen, Krankenhausaufenthalten oder einem geschwächten Immunsystem die Schule nur unregelmäßig oder gar nicht besuchen. Die Folgen sind nicht nur Einbußen der schulischen Fertigkeiten, sondern auch soziale und emotionale Schwierigkeiten (Kimmig, 2014; Pletschko, 2014). Das Fehlen von persönlichen Kontakten zu Lehrenden und Mitschüler*innen kann in vielen Fällen Gefühle von Einsamkeit und sozialer Isolation verursachen, und ein mangelndes Zugehörigkeitsgefühl begünstigt das Entstehen psychischer Folgeerkrankungen, einen geringen Selbstwert der Kinder, eine verminderte Lebensqualität und eine ungünstige Verarbeitung der Krankheit (Bishop \& Slevin, 2004; Forrest et al., 2011; Hopkins et al., 2013; Lum et al., 2017; McNeely et al., 2002; Yeo \& Sawyer, 2005). Um den negativen Folgeerscheinungen aufgrund des Fernbleibens vom Unterricht und sozialen Alltag in der Schule entgegenzuwirken, werden in den letzten Jahren zunehmend Telepräsenzsysteme, wie beispielsweise der Avatar AV1, als vielversprechender Ansatz diskutiert (Ahumada-Newhart et al., 2016; Gilmour et al., 2015; Soares et al., 2017).

\section{Avatar AV1}

Der Avatar ist ein kleiner Roboter, der anstelle der Kinder im Klassenraum platziert wird und mit einem Tablet von zuhause aus bedient werden kann. Ton kann in beide Richtungen übertragen werden, jedoch funktioniert die Videoübertragung nur in eine Richtung, sodass betroffene Kinder nicht zu sehen sind, aber umgekehrt die Mitschüler*innen sehen können. Die Kinder und Jugendlichen können über den Avatar kommunizieren, die Gesichtsausdrücke des Avatars bestimmen und so Gefühle der Klasse mitteilen. Der Avatar AV1 ist handlich und kann leicht getragen bzw. zu Schulausflügen mitgenommen werden. Übertragene Daten werden nicht gespeichert, da sowohl die Aufnahmefunktion des Avatars als auch des zugehörigen Tablets während des gesamten Einsatzes unterdrückt werden, die Übertragung erfolgt somit ausschließlich per Livestream. In Norwegen, Schweden, Deutschland und Großbritannien sind beispielsweise bereits 1200 dieser Avatare im Einsatz. 


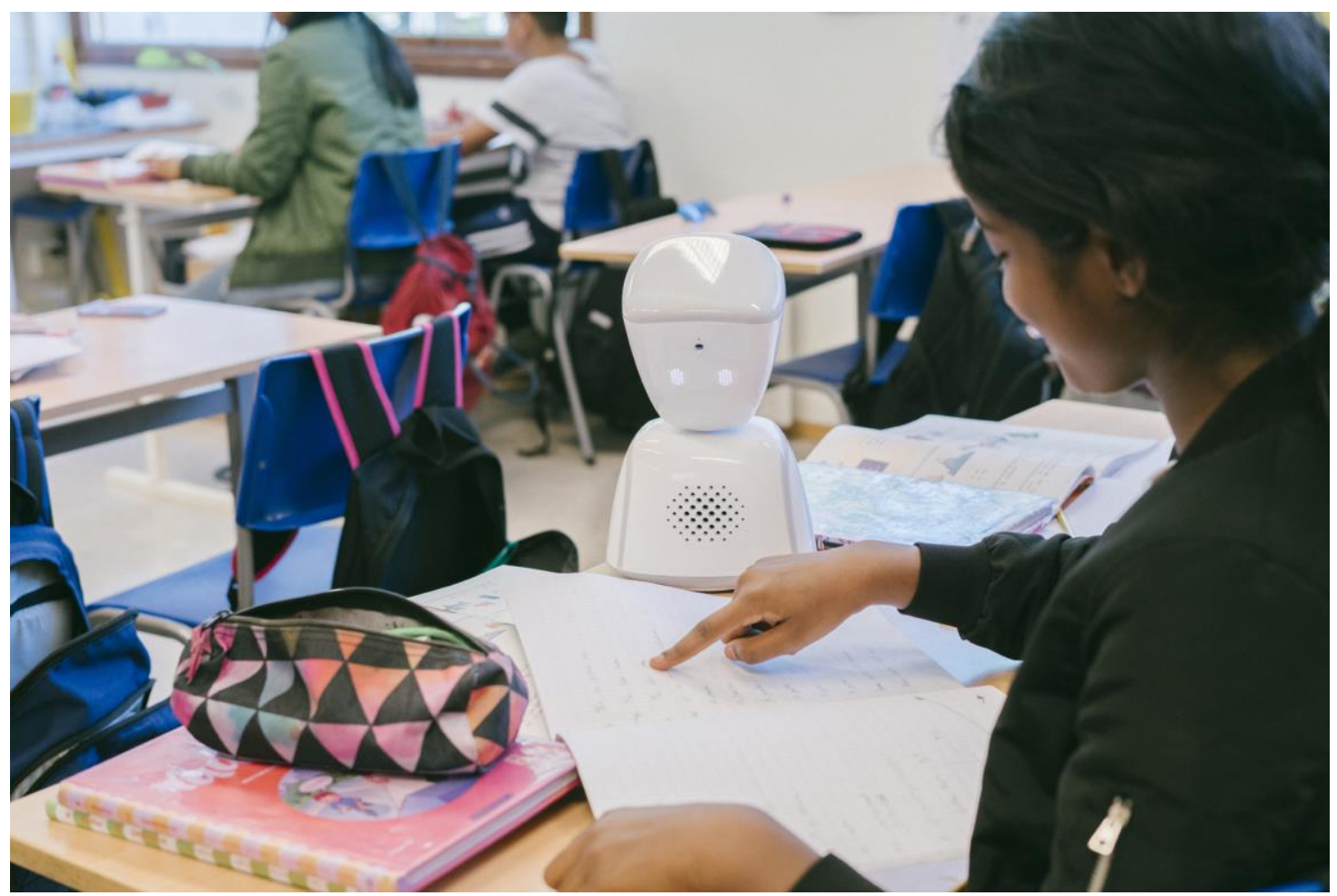

Abbildung 1: Avatar AV1 bei einer Gruppenarbeit | Foto: Estera K. Johnsrud

\section{Life happens wherever you are!}

Ein Projekt der Medizinischen Universität Wien in Kooperation mit der Firma Die Berater, der Heilstättenschule Wien sowie der Universität Klagenfurt hat sich zum Ziel gesetzt, den Einsatz des Avatars AV1 auch in Österreich zu ermöglichen. Der Fokus liegt dabei darauf, den möglichen negativen Folgeerscheinungen aufgrund des Fernbleibens des Unterrichts entgegenzuwirken und die Teilnahme am Regelschulalltag und die soziale Verbundenheit zu Mitschüler*innen mithilfe des Avatars aufrechtzuerhalten. Begleitend zum Einsatz der Avatare werden dessen Wirkungsweise und Effekte im Rahmen einer wissenschaftlichen Studie an der Medizinischen Universität Wien sowie der Universität Klagenfurt untersucht. Dabei soll herausgefunden werden, wie sich der Einsatz des Avatars AV1 auf die schulische Teilhabe, das Zugehörigkeitsgefühl zur Schule und Gefühle sozialer Isolation von chronisch kranken Kindern und Jugendlichen, die die Schule eine gewisse Zeit lang nicht besuchen können, auswirkt. Dabei sollen außerdem das Wohlbefinden, der Selbstwert der Kinder, die wahrgenommene Partizipation durch Eltern und Lehrer*innen, die Nutzungsdauer des Avatars und sozioökonomische Einflussvariablen erfasst werden. Zusätzlich wird in Kooperation mit der Universität Klagenfurt ein qualitativer Forschungsansatz verfolgt. Hierbei soll der Fokus im Rahmen einer Interviewstudie auf die Schüler*innen und ihre relevanten Umwelten (Eltern, Lehrer*innen, Mitschüler*innen) im schulischen Geschehen gelegt werden. Dabei soll vor allem der Frage 
nachgegangen werden, wie Schule mit dem Avatar erlebt wird und welche Chancen, Veränderungen und Hürden sich im schulischen System für die beteiligten Akteur*innen ergeben. Neben der Darstellung der Wirkungsweise des Avatars sollen Begleitangebote in Form einer Handreichung für Eltern und Lehrer*innen sowie ein Coachingangebot für Lehrer*innen im Umgang mit dem Avatar entstehen. Zusätzlich sollen die Ergebnisse in kindgerechter Form (z.B. Kurzzeichentrickfilm) dargestellt und ein Workshopangebot für Kinder- und Jugendliche entwickelt werden.

Das Projekt „Life happens wherever you are!“ wurde im November 2020 gestartet. Mittlerweile werden die Avatare bei etwa 20 Kindern in Wien, Niederösterreich, Salzburg, Vorarlberg, Tirol sowie in der Steiermark und dem Burgenland eingesetzt. Eine Genehmigung seitens des Bundesministeriums für Bildung, Wissenschaft und Forschung liegt für das gesamte Bundesgebiet vor. In Anbetracht der Anzahl chronisch erkrankter Kinder wird deutlich, dass hier, trotz der Anstrengungen der Projektpartner, auch weiterhin deutlicher Versorgungsbedarf besteht.

\section{Nutzen für Kinder und Jugendliche}

Chronisch kranke Kinder und Jugendliche bekommen mithilfe der Avatare, die als therapeutisches Hilfsmittel zum Einsatz kommen, die Möglichkeit, trotz fehlender körperlicher Anwesenheit weiterhin am Schulunterricht teilzunehmen und sozial eingebunden zu bleiben. Der Avatar selbst ist eine Innovation im Bereich der Telepräsenzsysteme, und obwohl bisher nur wenige Studien zu dessen Einsatz existieren, verdeutlichen diese, dass mit dem Design des Avatars ein gewisser "Coolheits"-Faktor verbunden ist und der Avatar von Mitschüler*innen aktiv in den Unterricht eingebunden wird (Culén, Borsting \& Odom, 2019; Weibel et al., 2020). Die Inklusion der Kinder bzw. Jugendlichen im schulischen Alltag und sozialen Gefüge der Klasse kann die Entwicklung und Erlangung bestimmter Fähigkeiten fördern, wobei sich dies im weiteren Verlauf positiv auf den Selbstwert sowie auf die sozialen Beziehungen der Kinder und Jugendlichen auswirken kann.

\section{Ausblick}

Ein besseres Verständnis für die Effekte der Nutzung des Avatars AV1 könnte dazu beitragen, die schulische Teilhabe von Kindern und Jugendlichen mit chronischen Erkrankungen in Zukunft nachhaltig zu verbessern. Darüber hinaus können Gefühle von sozialer Isolation und damit einhergehende, negative Folgeeffekte reduziert werden, indem Kinder frühzeitig und gezielt Unterstützung erhalten.

Geplante Publikationen im Rahmen des Projekts verfolgen des Weiteren das Ziel, Möglichkeiten für die Integration des Avatars AV1 in das Bildungssystem bzw. den Nutzen für ein breiteres Publikum aufzuzeigen und so die Situation für Schüler*innen mit chronischen Erkrankungen nachhaltig zu verbessern. 


\section{Literaturverzeichnis}

Ahumada-Newhart, V., Warschauer, M., \& Sender, L. S. (2016). Virtual inclusion via telepresence robots in the classroom: An exploratory case study. International Journal of Technologies in Learning, 23(4), S. 9-25.

Bishop, M., \& Slevin, B. (2004). Teachers' attitudes toward students with epilepsy: results of a survey of elementary and middle school teachers. Epilepsy \& Behavior, 5(3).

Culén, A. L., Børsting, J., \& Odom, W. (2019). Mediating Relatedness for Adolescents with ME. Proceedings of the 2019 on Designing Interactive Systems Conference - DIS '19, S. 359-371.

Forrest, C. B., Bevans, K. B., Riley, A. W., Crespo, R., \& Louis, T. A. (2011). School outcomes of children with special health care needs. Pediatrics, 128(2), S. 303-312.

Gilmour, M., Hopkins, L., Meyers, G., Nell, C., \& Stafford, N. (2015). School connection for seriously sick kids. Who Are They, How Do We Know What Works, and Whose Job Is It. Canberra, Australia: Australian Research Alliance for Children and Youth. https://www.aracy.org.au/publications-resources/command/download_file/id/331/filename/School-connection-for-seriously-sick-kids-full-report-web.pdf

Hopkins, L., Nisselle, A., Zazryn, T., \& Green, J. (2013). Hospitalised adolescents. A framework for assessing educational risk by. Youth Studies Australia, 32(1), S. 37-45.

Kimmig, A. (2014). Was hilft chronisch kranken Kindern in den allgemeinen Schulen. In E. Filtner et al. (Hrsg.), Chronisch Kranke Kinder in der Schule (S. 191-195). Stuttgart: Kohlhammer.

Lum, A., Wakefield, C. E., Donnan, B., Burns, M. A., Fardell, J. E., \& Marshall, G. M. (2017). Understanding the school experiences of children and adolescents with serious chronic illness: A systematic meta-review. Child: Care, Health and Development, 43(5), S. 645-662.

McNeely, C. A., Nonnemaker, J. M., \& Blum, R. W. (2002). Promoting School Connectedness: Evidence from the National Longitudinal Study of Adolescent Health. Journal of School Health, 72(4), S. 138-146.

Pletschko, T., Schwarzinger, A., Weiler, L., \& Leiss, U. (2015). Partizipationsskalen (PS 24/7). http://kinderklinik.meduniwien.ac.at/psychosoziales-team-der-neuroonkologie/klinik-projekte/fit-for-life/icfpartizipation-oder-marginalisierung/

Soares, N., Kay, J. C., \& Craven, G. (2017). Mobile Robotic Telepresence Solutions for the Education of Hospitalized Children. Perspectives in Health Information Management, 14 (Fall).

Weibel, M., Nielsen, M. K. F., Topperzer, M. K., Hammer, N. M., Møller, S. W., Schmiegelow, K., \& Bækgaard Larsen, H. (2020). Back to school with telepresence robot technology: A qualitative pilot study about how telepresence robots help school-aged children and adolescents with cancer to remain socially and academically connected with their school classes during treatment. Nursing Open, 7(4), S. 988-997.

Yeo, M., \& Sawyer, S. (2005). Chronic illness and disability. BMJ (Clinical Research Ed.), 330(7493), pp. 721-723. 


\section{Autor*innen}

\section{Thomas Pletschko, Dr.,}

ist Klinischer und Gesundheitspsychologe und hat sich in den Bereichen Kinder-, Jugend- und Familienpsychologie sowie Klinische Neuropsychologie spezialisiert. Er ist Leiter des Pediatric Brainfit Labs an der Medizinischen Universität Wien, wobei der Fokus seiner Forschung auf schulischer Reintegration sowie Partizipation liegt.

Kontakt: thomas.pletschko@meduniwien.ac.at

\section{Clarissa Pelzer, MSc MA,}

ist Klinische Psychologin und wissenschaftliche Mitarbeiterin an der Medizinischen Universität Wien im Bereich der pädiatrischen Neuroonkologie. Der Fokus ihrer Forschung liegt im Bereich des Einsatzes von Telepräsenzsystemen bei Kindern mit chronischen Erkrankungen.

Kontakt: clarissa.pelzer@meduniwien.ac.at

\section{Gerda Rockenbauer, BEd.,}

ist Pädagogin an der Heilstättenschule Wien und Projektkoordinatorin im Projekt „Virtual Classroom“. Sie war langjährige Direktorin einer Schule für körperbehinderte Kinder und Jugendliche.

Kontakt: gerda.rockenbauer@schule.wien.gv.at

\section{Agnes Turner, Assoc. Prof. ${ }^{\text {in }}$ Mag. ${ }^{\text {a }}$ Dr. ${ }^{\text {in }}$,}

ist Professorin für Pädagogik am Institut für Unterrichts- und Schulentwicklung an der Universität Klagenfurt. Sie lehrt im Bereich der Lehramtsausbildung und Weiterbildung von Lehrkräften und forscht u.a. zu emotionalen Aspekten beim Lernen und Lehren, mentalisierungsbasierter Pädagogik und Lernen und Lehren in einer digitalisierten Welt.

Kontakt: agnes.turner@aau.at 


\section{Martin Röhsner, Mag.,}

ist geschäftsführender Gesellschafter des privaten Bildungsträgers die Berater Unternehmensberatungs $\mathrm{GmbH}$ und selbst als Coach tätig. die Berater sind seit über 20 Jahren in der Aus- und Weiterbildung sowie Consulting am Markt und haben die Generalrepräsentanz der Avatare und verfügen über langjährige Expertise über OnlineLernplattformen sowie Telepräsenzsystemen.

Kontakt: m.roehsner@dieberater.com 